\title{
Giant coronary sinus aneurysm secondary to right coronary arteriovenous fistula leading to pseudo-mitral stenosis
}

Mario Gaudino ${ }^{1}$, Amedeo Anselmi ${ }^{1}$, Antonella Lombardo², Riccardo Marano ${ }^{3}$, Gianfederico Possati ${ }^{1}$

1Division of Cardiac Surgery, Catholic University, Rome, Italy
2Division of Cardiology, Catholic University, Rome, Italy
3Department of Bio-Sciences and Radiological Imaging, Catholic University, Rome, Italy

Submitted: 29 June 2010

Accepted: 27 August 2010

Arch Med Sci 2011; 7, 3: 533-535

DOI: 10.5114 /aoms.2011.23426

Copyright (c) 2011 \& Termedia

\author{
Corresponding author: \\ Mario Gaudino MD \\ Division of Cardiac Surgery \\ Catholic University \\ Largo A. Gemelli 8 \\ 00168 Rome, Italy \\ Phone: 0039-630154814 \\ Fax: 0039-630155881 \\ E-mail: mgaudino@tiscali.it
}

\begin{abstract}
We herein report a case in which a right coronary to coronary sinus arteriovenous fistula determined progressive aneurysmatic dilatation of the coronary sinus. Severe compression of the left atrium ensued. This led to a clinical and instrumental picture similar to that observed in severe mitral stenosis.
\end{abstract}

Key words: coronary sinus, coronary artery imaging, fistula (coronary artery).

\section{Introduction}

Coronary arteriovenous fistulas (CAF) are an uncommon finding in adult patients, and their clinical course is predominantly benign [1, 2].

We herein report an unusual case in which a CAF determined progressive aneurysmatic dilatation of the coronary sinus with consequent severe compression of the left atrium. This pathophysiological mechanism led to a clinical and instrumental picture similar to that observed in severe stenosis of the mitral valve.

\section{Case report}

A 78-year-old female patient was referred to our hospital due to a history of worsening dyspnoea and recent onset of atrial fibrillation. At physical examination, the patient appeared in a poor general condition, with severe effort dyspnoea (NYHA III), bilateral pulmonary rales and peripheral oedema. A systo-diastolic murmur could be clearly heard in the precordial area. Transthoracic echocardiography revealed a large anecogenic mass (diameters: $5 \mathrm{~cm} \times 7 \mathrm{~cm} \mathrm{max)}$ at the level of the postero-lateral left atrial wall. This determined severe compression of the atrial chamber, increased left atrial and pulmonary pressure and evidence of blood stasis within the left atrium (Figure 1). The observed physiopathology closely resembled the picture of severe mitral stenosis, although both the mitral valve and subvalvular apparatus appeared morphologically normal. Due to the poor general condition of the patient a cardiac tumour or, less likely, a pericardial cyst was 


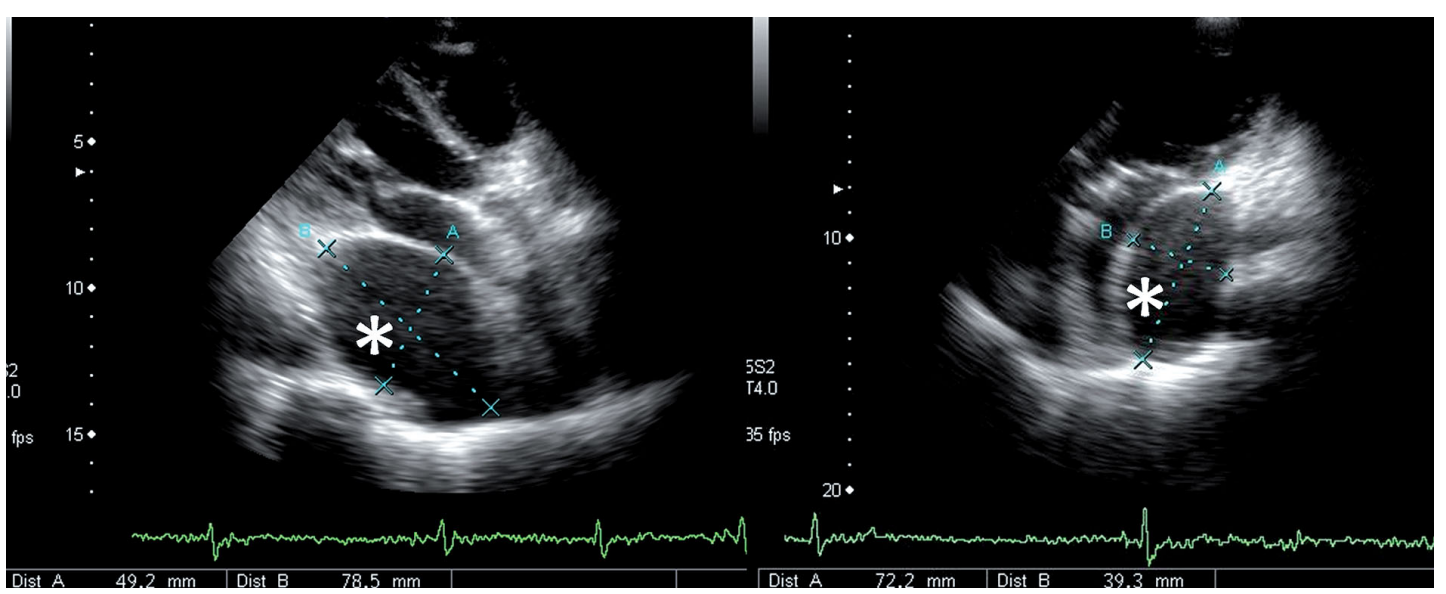

Figure 1. 2D-Echocardiographic image obtained from parasternal long-axis view. White asterisk: giant $(49.2 \mathrm{~mm} \times$ $\times 78.5 \mathrm{~mm}$ ) coronary sinus as a result of the fistula connecting the right coronary artery with the coronary sinus. The significant compression of this mass on the left atrium is appreciable, with ensuing distress of the left ventricular filling

$L V$ - left ventricle, $L A$ - left atrium

suspected. Contrast-enhanced CT scan of the chest revealed a right coronary-coronary sinus fistula with giant dilatation of the right coronary artery (mean diameter $20 \mathrm{~mm}$ ) (Figure 2) and massive aneurysmal dilatation of the coronary sinus (maximum diameters $11.2 \mathrm{~cm} \times 8 \mathrm{~cm}$ ). Diffuse calcification was evident within the wall of the aneurysmatic coronary artery. Moreover, the aneurysm of the coronary sinus led to a marked compression of the left atrium, which showed a semilunar shape and was reduced to a volume of $62 \mathrm{ml}$. Coronary angiography confirmed the diagnosis and demonstrated severe pulmonary hypertension (mean pulmonary arterial pressure $70 \mathrm{mmHg}$ ) and increased left atrial pressure.

On the basis of the severe clinical symptoms and of the impossibility to achieve satisfactory haemo- dynamic compensation with medical therapy, surgical treatment was decided.

After femoro-caval venous cannulation, cardiopulmonary bypass was established and the ascending aorta cross-clamped. Administration of antegrade blood cardioplegia led to filling of the coronary sinus with bright red blood, thus confirming the location of the distal orifice of the fistula. The aneurysmatic coronary artery was opened along its entire length and suture-closed both proximally and distally. A saphenous bypass graft was then performed on the distal posterior descending artery, which appeared of normal size. The aneurysmatic coronary sinus was incised longitudinally, the distal orifice of the fistula was located and closed by direct suture, and the free

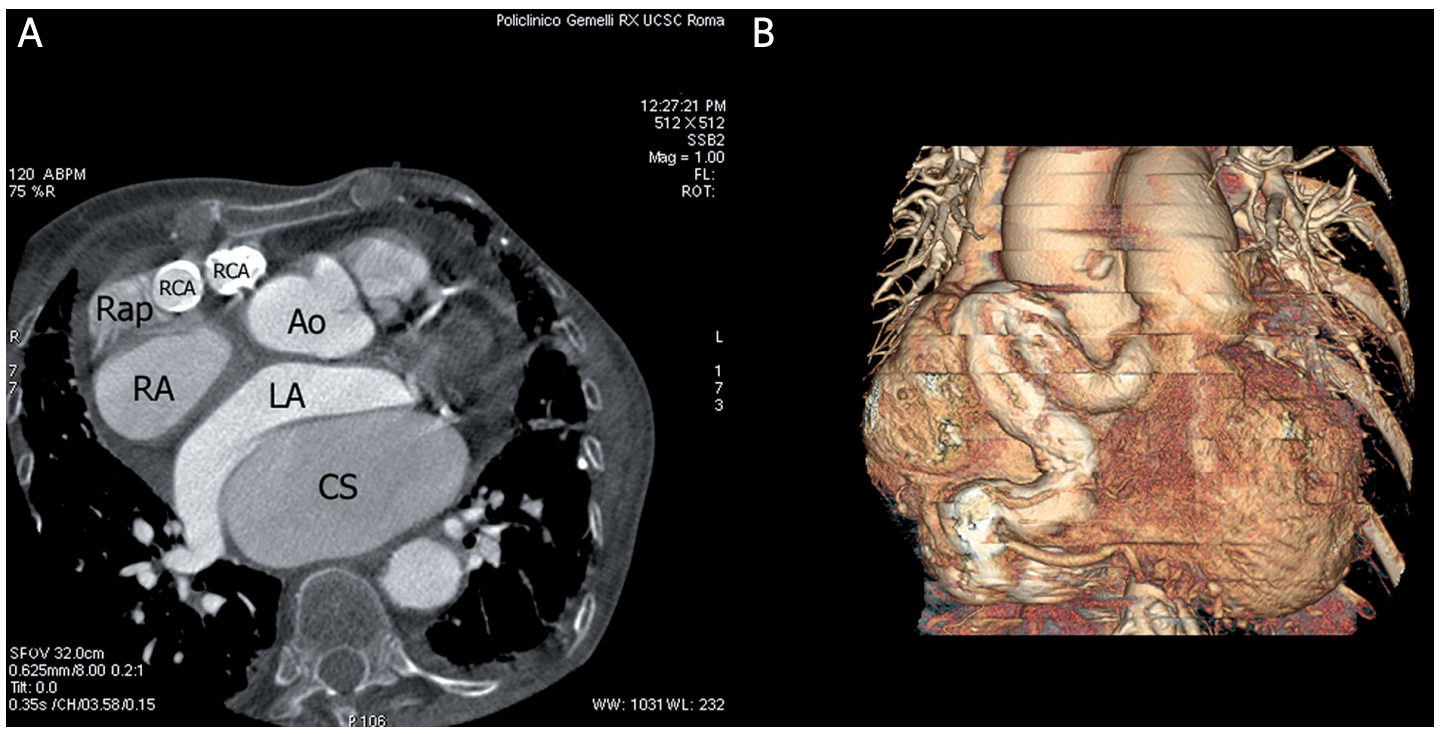

Figure 2. A - the CT axial image shows the conspicuous enlargement of the coronary sinus, with antero-superior displacement and compression of the left atrium. B - volume-rendered 3D reconstruction showing the tortuous course of the ectatic and calcified right coronary artery

Ao - aorta, CS - coronary sinus, $L A$ - left atrium, $R A$ - right atrium, $R C A$ - right coronary artery 
wall of the coronary sinus was plicated. The postoperative course required prolonged inotropic and respiratory support. The patient was finally discharged from intensive care on the $21^{\text {st }}$ postoperative day.

\section{Discussion}

Coronary arteriovenous fistulas are an uncommon finding in adult patients. In the majority of cases they are congenital in origin, and result from the persistence during embryonic development of junctions of primordial epicardial vessels with intramyocardial sinusoidal circulation. However, rare non-congenital fistulas (iatrogenic, post-traumatic) have also been described. The prevalence of CAF is reported at about $0.1 \%$ of all routine cardiac angiographies [3].

Rarely, CAF are complicated by the development of a giant coronary artery aneurysm. To date, less than forty cases of CAF + giant coronary aneurysm have been described in the English literature. In the majority of cases, the right coronary artery is involved and the most common draining sites are the pulmonary artery and the right atrium. Drainage into the coronary sinus has been reported in only seven cases. The association of CAF, giant coronary aneurysm and coronary sinus aneurysm is even rarer [1-4].

This type of malformation usually remains asymptomatic. Surgical intervention is required only rarely, and the main indications for surgery are heart failure (due to the chronic left-to-right shunt), myocardial ischaemia (due to coronary steal) and risk of acute rupture. Mechanical compression of other cardiac structures (namely the left atrium) leading to "pseudo-mitral stenosis" as in this case has not been described before.

The only similar case has been recently described by El Watidy et al. [5]: the aneurysmatic coronary sinus was thrombosed in this patient, and caused severe mitral and tricuspid regurgitation, presumably from annular distortion and dilatation.

In our case the coronary sinus aneurysm was not thrombosed, but led to severe compression of the left atrium. The compression was clearly evident in both echocardiography and the CT scan, and the left atrial chamber appeared semilunar in shape with reduced volume and evidence of flow stasis (see Figures). This in turn led to a clinical and haemodynamic picture almost perfectly identical to that of severe mitral stenosis (despite the fact that the mitral valve and subvalvular apparatus appeared morphologically normal).

The surgical risk for the correction of these rare entities may be considerable. Perioperative myocardial infarction has been suggested as a potential complication of the ligation of a CAF $[5,6]$. Nonetheless, the patients surviving surgery apparently have a favourable prognosis, as outlined in our case and in the literature [5]. However, longitudinal follow-up studies are currently unavailable to formally address this issue.

In conclusion, we describe an unusual case of right coronary to coronary sinus arteriovenous fistula associated with aneurysmatic dilatation of both the coronary artery and the coronary sinus. These features led to severe interference with the left atrial and mitral function and to a picture of "pseudo-mitral stenosis". It is noteworthy that CAF with aneurysmatic dilatation of the receiving cardiac chamber can lead to mechanical interference with atrioventricular valve function and pseudo-valve stenosis.

\section{References}

1. Gandy KL, Rebeiz AG, Wang A, Jaggers JJ. Left main coronary artery-to-pulmonary artery fistula with severe aneurismal dilatation. Ann Thorac Surg 2004; 77: 1081-3.

2. Nakahira A, Sasaki Y, Hirai H, et al. Rupture of aneurysmal circumflex coronary artery into the left atrium after ligation of its arteriovenous fistula. Circ J 2007; 71: 1996-8.

3. Davidavicius G, Subkovas E, Abraitis V, Zabukas A, Beniusis V. Coronary anomalies: single center experience. Semin Cardiol 2004; 10: 208-13.

4. Komoda S, Komoda T, Ivanitskaia-Kuehn E, Dreysse S, Pasic M, Hetzer R. Giant aneurysm of the right coronary artery and fistula to the coronary sinus. Gen Thorac Cardiovasc Surg 2010; 58: 78-81.

5. El Watidy A, Ismail H, Calafiore AM. Surgical management of right coronary artery coronary sinus fistula causing severe mitral and tricuspid regurgitation. Interact Cardiovasc Thorac Surg 2010; 10: 110-2.

6. Hoendermis E, Waterbolk T, Willems T, Zijlstra F. Large common left and right coronary to coronary sinus fistula. Interact Thorac Cardiovasc Surg 2006; 5: 778-9. 